\title{
Circuit
}

Musiques contemporaines

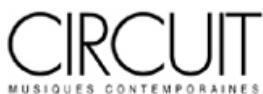

\section{Compositeurs chinois en Europe : entretiens avec Chen Qigang et Wen De-Qing}

\section{Nicolas Donin}

Volume 12, numéro 3, 2002

La route de soi

URI : https://id.erudit.org/iderudit/402001ar

DOI : https://doi.org/10.7202/402001ar

Aller au sommaire du numéro

Éditeur(s)

Les Presses de l'Université de Montréal

ISSN

1183-1693 (imprimé)

1488-9692 (numérique)

Découvrir la revue

Citer ce document

Donin, N. (2002). Compositeurs chinois en Europe : entretiens avec Chen Qigang et Wen De-Qing. Circuit, 12(3), 9-34. https://doi.org/10.7202/402001ar

\section{Résumé de l'article}

Entretiens réalisés à Paris, en février 2002, avec Chen Qigang et Wen De-Qing, compositeurs chinois nés dans les années 1950 et vivant maintenant respectivement à Paris et à Genève. Tous deux sont partis en Europe passée la trentaine; leurs parcours n'en sont pas moins très différents l'un de l'autre. Nicolas Donin les questionne sur leur cheminement et leur musique, s'efforçant de leur faire mettre en lumière la part de contingence et la part de volonté dans les ruptures - géographie et esthétique - qui ont jalonné leur vie de musicien. 


\title{
Compositeurs chinois en Europe : entretiens avec Chen Qigang ef Wen De-Qing
}

\author{
Nicolas Donin
}

Les deux entrefiens qui suivent donnent la parole à des compositeurs chinois nés dans les années 1950. Tous deux sont partis en Europe passée la trentaine; leurs parcours n'en sont pas moins très différents l'un de l'autre, à quelques années d'intervalle. Je me suis efforcé, dans la mesure du possible, de leur faire dévoiler la part de contingence ef la part de volonté dans les ruptures - géographique et esthétique allant en général de pair, ici - qui ont jalonné leur vie de musicien.

J'ai renoncé à fournir une liste d'œuvres représentatives pour chacun d'eux, ce qui n'aurait pas eu grand sens pour le lecteur canadien. Je renvoie d'une part à leurs éditeurs (Gérard Billaudot (Paris) pour Chen', Swiss Music Edition [SME] (Lucerne) pour $\mathrm{Wen}^{2}$ ), d'autre part à leurs disques monographiques:

Chen Qigang. Poème lyrique II, Feu d'ombres, Yuan, Yi, Ensemble instrumental de Ville d'Avray, Orchestre philharmonique de Radio France, CD MFA REM $311223,1993$.

Wen Deqing (Le souffle, Quatuor à cordes $n^{\circ} 1$, Wu, Complainte, Ji I \& Ji III. Ensemble Contrechamps, Giorgio Bernasconi, CD Stradivarius STR 33471, 1996.

Les deux entretiens ont été réalisés à Paris au début de février 2002, dans l'ordre inverse de leur publication ici 4 ef 6 février pour Wen, 10 février pour Chenl. J'ai voulu initialement poser la même question, pour mieux voir dévier chaque dialogue; en revanche, je ne m'attendais pas à ce que les deux entretiens s'achèvent de façon quasi similaire...

1. L'adresse du site internet de Chen est http://qgchen.free.fr/

2. http://unw.musicedition.ch/composers/ 107e.htm 


\section{« Assimiler la musique contemporaine m'a permis de mesurer le décalage entre elle et moi. »}

\section{Entretien avec Chen Qigang.}

À brûle-pourpoint, Chen Qigang commence à me parler d'un fait récent de la vie musicale parisienne, auquel lui comme moi avons assisté : la façon dont Tan Dun s'est fail huer par une partie du public lors de l'ouverture du festival Présences 2002. Pendant une vingtaine de minutes - je n'ai pas encore installé le matériel pour enregistrer - nous discutons de la signification de ce micro-événement. Chen est choqué par les réflexes du public l'exécution du concerto pour violoncelle de Tan Dun par Anssi Karttunen ${ }^{2}$ a été interrompue entre deux mouvements par des huées et des sifflements ambigus : certains croyaient que c'était la fin de l'œuvre, d'autres non; certains voulaient applaudir la virtuosité des interprètes, d'autres huer le compositeur - qui dirigeait l'orchestre; etc.). Pourquoi contesteraiton à un créateur le droit de s'exprimer en lui signifiant si bruyamment son mécontentement? Je réponds à Chen qu'il n'existe malheureusement pas de moyen plus raffiné pour exprimer une désapprobation profonde. Chen fait alors une comparaison : si un compositeur français contemporain venait en Chine diriger sa musique, le public n'y comprendrait assurément rien, mais ne porterait pas de jugement à l'emporte-pièce ; il applaudirait, tièdement s'il n'a pas aimé, mais il applaudirait. Tan Dun a rapporté à Chen Qigang qu'au cours d'une tournée qui l'avait mené dans une dizaine de pays, la France était le seul endroit où sa musique avait suscité une réaction semblable. Chen dit que, par certains côtés, la France est incapable d'écouter une production qui échappe aux normes tacites définies depuis les années 1970. M'apercevant que je $\mathrm{m}$ 'apprête à répondre comme si c'élait moi l'interviewé, j'allume mon microphone et nous commençons l'entretien:

Nicolas Donin - Vous souvenez-vous de la première fois que vous avez entendu de la musique?

Chen Qigang - Quand j'étais enfant? Bien sûr. II faut d'abord rappeler que je suis issu d'une famille d'artistes. Ma mère est musicienne; ma sœur a commencé le piano avant moi; mais ce qui me touche le plus, c'est la musique traditionnelle parce que mon père - qui était peintre - en était un grand amateur et l'avait enseignée dans sa jeunesse. Souvent, quand il rentrait à la maison, il jouait du erhu ["violon chinois", vièle à deux cordes]. N'oubliez pas qu'en Chine, pour être reconnu comme peintre ou comme lettré, on doit posséder des connaissances en musique, savoir jover aux échecs, pratiquer la calligraphie et peindre : ce sont les
2. Crouching Tiger Concerto, pour violoncelle, vidéo et orchestre. Festival Présences, concert du mercredi 30 janvier 2002, salle Olivier-Messiaen, Maison de Radio France, Paris. 
quatre qualités indispensables. Donc j'ai toujours entendu de la musique traditionnelle chez moi.

Nicolas Donin - Mais votre sœur, si elle jouait du piano, devait bien jouer de la musique...

Chen Qigang - ... occidentale, en effet, et ça reste choquant pour moi. Un accord parfait... c'est déjà tout autre chose. Un enfant qui commençait à apprendre le piano à cette époque le faisait à travers des accords parfaits et des rythmes réguliers assez clairs : cela donne une tout autre image de la musique. Au début, les deux cultures musicales étaient amalgamées pour moi.

Nicolas Donin - Vous n'avez donc pas de souvenirs distincts, liés soit à l'une soit à l'autre. Mais je suppose que cet amalgame n'a pas pu perdurer au moment de vos études.

Chen Qigang - Quand j'étais à l'école primaire, mon père voulait que je devienne acteur de l'opéra de Pékin, et il m'a enseigné le chant. Mais comme ma sœur était pianiste, j'ai voulu aller à l'école de musique, pour apprendre la musique occidentale. À l'époque, néanmoins, il n'y avait pas de distinction nette entre les deux cultures, même pour un jeune Chinois: beaucoup de chansons comme Frère Jacques sont pratiquées en Chine, mais considérées comme chinoises. L'origine de chaque musique n'est pas nécessairement connue ou prise en considération. Peut-être cette confusion estelle liée à cent cinquante ans de problèmes d'occupation par les Occidentaux ef les Japonais. Quoi qu'il en soit, c'est seulement au Conservatoire que j'ai commencé à comprendre la différence.

Nicolas Donin - En abordant la composition?

Chen Qigang - Non, je suis entré au Conservatoire à 13 ans - comme instrumentiste. J'ai été clarinettiste pendant quatorze ans, dont cinq comme clarinette solo d'un orchestre symphonique, ce qui m'a permis de connaitre tous les instruments, de faire des transcriptions et arrangements avec des amis instrumentistes; c'est dans ce contexte que j'ai commencé à composer, c'està-dire avant même d'entrer au Conservatoire supérieur pour être compositeur. De sorte qu'au Conservatoire, j'avais déjà une expérience du travail d'orchestre et de la composition pour orchestre, contrairement à la plupart des autres étudiants. C'est une des raisons pour lesquelles je suis si sensible au son et à la combinaison des différents timbres.

Nicolas Donin - Est-ce à cette époque que vous avez rencontré Tan Dun?

Chen Qigang - Il est plus jeune que moi - nous avons six ans d'écart -, donc pendant la Révolution culturelle il était encore enfant et moi, i'avais 14 ans. Nous sommes entrés en même temps dans la section composition du Conservatoire, en 1978, mais il n'avait pas un passé de musicien comme moi. Et cela s'entend, ainsi que chez Qu Xiaosong et Guo Wenjing qui n'avaient pas non plus de formation 
avant le Conservatoire : si Tan Dun est si audacieux pour certaines choses, c'est qu'il n'est pas vraiment profondément influencé par la culture musicale occidentale.

Nicolas Donin - Il a moins à perdre, d'une certaine manière?

Chen Qigang - En quelque sorte. II ne sent pas autant le besoin de respecter le cadre. Tandis que moi, j'avais neuf ans de formation à l'école secondaire du Conservatoire.

Nicolas Donin - Si cette formation de clarinettiste est déterminante, alors ce n'est sûrement pas un hasard si une de vos premières œuvres importantes créées en France a été Yi pour clarinette et quatuor à cordes [1986]?

Chen Qigang - En effet. Ce n'est pas la première œuvre que j'ai écrite en France, mais c'est la première à avoir marqué quelque chose. J'étudiais alors avec Messiaen, et c'est la première œuvre qu'il ait beaucoup appréciée. Dès qu'il a vu les premières pages, il a dit: "Ha ! ça c'est bien! " Qu'est-ce qui était bien? Je ne le savais pas; mais après, j'ai compris que c'était d'abord lié à l'impression visuelle faite par la partition : le dessin, la façon d'écrire les notes, la façon dont étaient dispersées les notes sur une page, le contraste entre les lignes, etc. C'est la première fois que c'est rentré dans ma tête. Avant, je pensais tout à fait spontanément à exprimer ce que j'avais envie de dire, mais là j'ai été vraiment influencé par la musique contemporaine. Je n'avais soudain plus besoin du piano pour écrire - ça, je crois, c'est plutôt un tort, car si on n'a pas une référence, comment peuton écrire une musique contrôlée et souhaitée?

Nicolas Donin - Mais la particularité de la musique qu'on souhaite, c'est justement qu'elle n'existe pas encore!

Chen Qigang - Certes, elle ne peut pas exister préalablement, mais il faut avoir de l'expérience. Cette expérience-là, on ne l'acquiert pas en jetant sur le papier n'importe quels signes et en disant, à l'écoute du résultat : "Ceci, c'est ce que j'ai voulu; cela, ce n'est pas ce que j'ai voulu; etc. "Or à l'époque, j'étais à ce niveau. Je ne savais pas ce que je voulais, et j'ai suivi certaines théories.

Nicolas Donin - Messiaen a-t-il valorisé cette dissociation de l'écriture et de la perception?

Chen Qigang - Tout à fait; mais je crois qu'il avait raison : par rapport à ce que j'avais fait jusque-là, une étape était franchie. II a dit: "Vous entrez dans un autre monde, complètement différent. »C'était inévitable, et historiquement c'est une très belle chose : quand la pièce a été jouée, Marc Texier (qui travaillait alors à France Musique) m'a téléphoné - alors que nous ne nous connaissions pas - et il m'a dit : "Vous avez fait une excellente pièce. " Moi, je ne le savais pas. Je n'avais pas fait exprès. Avec le temps, j'ai réalisé que ce n'était pas moi. Il y avait une part de moi, bien sûr : les élans, les liaisons entre chaque partie, c'était moi. Mais le langage, c'était plutôt Ligeti, ou... 
Nicolas Donin - ... Ferneyhough?

Chen Qigang - Non, pas vraiment, il n'était pas encore très la mode. C'est venu plutôt en 1987, à Darmstadt en 1988...

Nicolas Donin - Vous étiez à Darmstadt en 1988. C'est la seule fois que vous y êtes allé?

Chen Qigang - J'y suis allé deux fois. Comme j'ai eu un prix en 1988, j'ai été invité en 1990. En 1990, déjà, j'ai commencé à comprendre que ce n'était pas mon monde. Mais avant, j'ai voulu être dans la mode jusqu'au bout - dans ce système contemporain, dans la peau des Européens. Ce n'était même pas par manque de courage : je n'avais pas conscience de ce qui m'appartenait ou pas, de ce qui était bien ou de ce qui ne l'était pas.

Nicolas Donin - Mais lorsque vous avez quitté la Chine, cela partait bien d'un désir, de l'idée que c'était cette musique-là qu'il allait falloir écrire?

Chen Qigang - Oui, mais inconsciemment. On disait que l'Europe était le meilleur endroit puisque c'était le lieu de naissance de cette musique éprouvée par le temps. Après, j'ai compris que cela n'était pas si vrai. Mais à ce moment-là, je pensais - Tan Dun aussi, Guo Wenjing et les autres aussi le pensaient - que la vérité se trouvait là-bas. Il y a une chose que je ne regretterai jamais, c'est d'avoir eu Messiaen comme professeur.

Nicolas Donin - Comment s'est passée la rencontre avec Messiaen?

Chen Qigang - C'est comme une anecdote. Messiaen, à l'époque, c'était le père spirituel de la musique française, un modèle vivant pour le monde entier. Quand je suis arrivé à Paris, lors d'un échange culturel franco-chinois, un copain m'a dit : "ll faut choisir. Choisir le Conservatoire de Paris ou l'université, choisir tel ou tel professeur", etc. Pour nous, à l'époque, le diplôme comptait beaucoup, mais une fois arrivés en France, nous avons réalisé qu'il n'y avait pas de doctorat interne au Conservatoire, pas de diplôme universellement valable. Soit on entrait à la Sorbonne ou dans une autre université pour avoir ce genre de diplôme, soit on continuait à étudier la composition mais sans diplôme. Lors d'une discussion, un ami m'a dit: "Si, avec un professeur, même sans diplôme, vous pouvez avoir un résultat d'étude qui vaut mieux qu'un diplôme, dans ce castlà vous pouvez abandonner le diplôme. "En France, deux noms seulement correspondaient à cela : Messiaen, Boulez. Boulez n'enseignait pas, Messiaen n'enseignait plus. L'ami m'a dit : "Pourquoi ne pas leur écrire une demande? " Je n'avais pas l'adresse. Un jour, par hasard, dans la rue à Bordeaux, nous discutions avec un vieil homme à vélo, et il nous a dit : "Mon beau-fils est l'élève d'Yvonne Loriod." II a téléphoné pour avoir l'adresse, j'ai écrit une lettre en chinois que mon ami a traduite, et on l'a envoyée. Un mois et demi après, dans ma boîte aux lettres, j'ai vu la réponse de Messiaen. Avant même de l'ouvrir, ma main tremblait : je savais que quelque chose m'arrivait. Messiaen disait : "Je suis très ému de votre lettre si chaleureuse, 
mais avant de vous répondre d'une façon absolue, j'aimerais vous rencontrer, voir ce que vous avez fait, etc. Je serai de retour en France vers octobre - ça se passait en août, en plein été - si vous voulez je vous donne rendez-vous le 22 octobre à 17 heures, vous venez à telle adresse avec vos partitions et enregistrements. On va discuter ensemble. " Ça, c'est Messiaen... Même moi aujourd'hui, quand il y a une demande de ce genre, je ne réponds pas. Et lui, il avait répondu! Il avait des milliers de demandes de ce genre. Et il m'a répondu. C'était un miracle.

J'avais deux mois devant moi : je ne connaissais pas la musique contemporaine, je ne connaissais pas vraiment Messiaen, je ne savais pas quel homme il était, si j'aimais sa musique... Je ne connaissais pas l'histoire de la musique trançaise, ni la langue, etc. Ces deux mois étaient cruciaux. J'ai fait tout ce que je pouvais pour préparer le rendez-vous : apprentissage de la langue, connaissance de I'histoire de la musique française, connaissance de tout ce qu'il avait fait thomme, musique, attitude, position socialel, et tentative d'écrire une pièce pour piano. L'ami qui avait traduit la lettre pour moi m'a beaucoup aidé; on a fait des simulations de l'entretien où il jouait le rôle de Messiaen. Le 22 octobre, je suis allé chez lui. Ce premier rendez-vous a duré quatre heures. Cela montre qui était cet homme, combien il était ouvert, quelles étaient ses qualités humaines (ne serait-ce que la patience avec laquelle il essayait de comprendre quelqu'un qui parlait à peine français). J'essayais d'être aussi naturel que possible. Au début, il avait dit une phrase qui aurait pu me faire perdre tout espoir : "Je suis trop âgé, pour vous faire connaître ce qui se passe actuellement il vaudrait mieux un professeur plus jeune, je peux vous recommander à un très bon professeur du Conservatoire», etc. J'ai fait comme si je n'avais rien entendu, j'ai continué la conversation. Et à la fin, dans les dernières minutes, il a demandé à Yvonne Loriod de venir et lui a dit : "Qu'en pensez-vous? j'ai envie de l'accepter. " Elle a dit : "Mais... si vous voulez! » [silence; rires]. Aussitôt il a rédigé une lettre pour le CROUS [Centre régional des œuvres universitaires et scolaires] de Paris disant qu'il avait accepté de me donner des leçons plusieurs fois par mois, et précisant divers détails matériels. Quand je suis sorti de chez lui, je savais que quelque chose commençait.

La première leçon a été très marquante pour moi : c'était la première fois de ma vie que quelqu'un me disait : "Soyez vous-même, c'est le seul espoir pour un compositeur, il ne faut pas trop s'occuper de l'idée des autres ou de la mode. Si un jour j'entends une musique et que, sans aucune explication, je sais que c'est de vous, alors vous aurez réussi. " En Chine, on recevait un enseignement tout à fait contraire. On nous disait d'écrire pour les autres, pour que les autres puissent comprendre : être au service des autres. Messiaen a dit : "Quel autre? Vous parlez des soldats, des ouvriers et des paysans, mais auquel en particulier vous adressez-vous? Chaque personne a son goût, vous ne pourrez jamais satisfaire tout le monde. Faites ce que vous voulez faire, et dans ce cas, il y aura des gens pour vous comprendre, à un niveau beaucoup plus profond. ” Bien sûr, facile à dire, difficile à 
faire. II y a une pression omniprésente des collègues, des professeurs, des institutions, des ensembles de musique contemporaine. II y a une sélection naturelle des œeuvres, et si vous faites quelque chose à part, les gens vous disent que c'est nul... J'ai d'abord eu une période d'assimilation - copier les chefs-d'œuvre - ce qui m'a permis, je l'ai compris plus tard, de mesurer le décalage entre cette musique et moi. Il faut beaucoup de temps. C'est en 1990 que j'ai commencé à imaginer des choses intéressantes comme Poème Lyrique II.

Nicolas Donin - Pendant combien de temps avez-vous vu Messiaen régulièrement? Chen Qigang - Quatre ans, entre 1984 et 1988. Selon son secrétaire, j'étais le chouchou de la famille. Je ne payais pas les leçons - c'est le CROUS qui réglait le professeur - mais après chaque trimestre ou semestre, Messiaen me demandait toujours: "Comment ça va, la vie, avez-vous des difficultés? " et il me remettait toujours une enveloppe en me disant d'achetez quelque chose avec. C'est toujours émouvant quand je repense à un tel professeur. Maintenant, quand je travaille avec un jeune, je ne peux pas oublier ça; il faut aider les jeunes à trouver leur chemin ef leur donner les moyens de continuer. Ça c'est un devoir, je crois - ça l'est devenu.

Nicolas Donin - Quand cette période s'est progressivement terminée, à l'époque où vous avez composé Yuan pour grand orchestre [1987-1988], il y avait toute une génération d'élèves de Messiaen qui avait une influence considérable: les spectraux. Avez-vous eu besoin de vous situer par rapport à eux?

Chen Qigang - J'étais très ami avec Marc-André Dalbavie. Hurel et moi étions ensemble dans la classe d'lvo Malec. Quant à Gérard Grisey, nous avons été ensemble dans une fondation [La Napoule] pendant trois mois; nous avons échangé beaucoup d'idées. Malgré la différence culturelle, je suis toujours en bon rapport avec Dalbavie; il a une liberté d'expression très différente des autres. Humainement, aucune personne n'est ennemie; on est tous égaux et on pourrait discuier. Cela n'empêche pas d'avoir des idées indépendantes. Je respecte les autres, mais j'ai parfois l'impression que les autres ne me respectent pas. Je ne suis vraiment pas de cette école.

Nicolas Donin - Mais dans Yuan, par exemple, on sent que vous en tenez compte - sans pour autant qu'il s'agisse d'une influence ou d'une appartenance.

Chen Qigang - \|l faut plutôt se référer à la musique de George Benjamin, dont j'ai beaucoup apprécié les œuvres de jeunesse - après, un peu moins, lorsqu'il a été influencé par la musique spectrale ou par l'IRCAM, c'est pour moi très différent. Mais ça n'empêche pas la communication - on s'écrit et on se voit régulièrement.

Nicolas Donin - C'est par Messiaen que vous l'avez rencontré?

Chen Qigang - Oui. C'est un autre chouchou de Messiaen, qui en parlait sans arrêt. $\grave{A}$ un moment on était un peu jaloux, surtout lui parce qu'il aurait dû être le dernier élève de Messiaen et que j'avais en quelque sorte pris cette place! [...] 
Nicolas Donin - Pour en revenir à Yuan, je me suis demandé si la musique polonaise (Lutoslawski en particulier) n'avait pas influencé votre travail, notamment pour la gestion des climax et l'écriture des houles orchestrales en aléatoire contrôlé (procédé que vous utilisez encore fréquemment).

Chen Qigang - Un peu, mais pas en profondeur. Je suis allé en Pologne en 1992 ; j'ai discuté de Yuan avec des Polonais, à Darmstadt en 1990. Pour eux, cette pièce était un peu exagérée : il y a un climax trop fort par rapport aux proportions. Pour moi, l'écriture de cette pièce introduisait un peu ce que je conservais de la Chine au sein de l'écriture complexe que j'avais intériorisée - par exemple dans la façon de traiter les trompettes, dans les mélodies, etc. Mais la plus forte influence qui s'est exercée sur cette pièce, je crois que c'est Ligeti, puis Britten rien à voir! - puis Ravel, Debussy et Messiaen. Presque tous les accords suivent le principe de construction créé par Messiaen. C'est la pièce où j'ai investi le plus d'énergie jusqu'à maintenant. Je prenais près d'une semaine à écrire une page. Au bout du compte, était-ce utile? C'est une autre question; mais à l'époque j'ai voulu faire la chose la plus complexe, la plus riche et la plus sensible possible. Tout ça à mettre ensemble, ce n'était pas facile, donc cela a pris un temps fou. Je ne le ferais plus. Plus tard je me suis apperçu que j'aurais pu obtenir le même résultat en écrivant beaucoup plus simplement. II faut le faire une fois pour comprendre; après, on n'est plus la même personne.

Nicolas Donin - À cette époque-là, vos œuvres portaient très souvent un titre chinois el leur contenu était, disons, musique-contemporaine-française. À quel moment situez-vous le retour audible de la Chine dans votre musique?

Chen Qigang - II y aurait déjà Voyage d'un rêve [1987; pour flûte, harpe, percussions et trio à cordes], avant Yuan. Quand lvo Malec a entendu Voyage d'un rêve, il a dit: [ton de reproche effaré] "Il ne faut pas faire ça!"; tandis que Ferneyhough, paradoxalement, a dit "Qui dit que c'est interdit? non, ça marche bien!", et c'est même lui qui a décidé de me donner un prix, à Darmstadt, pour cette pièce-là. Je ne savais pas qui avait raison, j'avais beaucoup de mal à comprendre!

Nicolas Donin - Teniez-vous compte du public? Commenciez-vous à vous dire qu'il y avait un public pour votre musique, avec lequel vous communiquiez?

Chen Qigang - Non, car ça s'est fait naturellement. C'était un succès chaque fois, donc je me suis dit : eh bien, il faut continuer!

Nicolas Donin - Et Poème Lyrique II, ça été un encore plus gros succès.

Chen Qigang - Oui, et je ne sais pas pourquoi. Ils ont même fait un film dessus, en Hollande [De Sprong, par Sylvia Stoetzer et Mirjam Van Veelen]. C'est ma pièce la plus jouée (notamment grâce à la formation instrumentale, qui est commode) avec San Xiao [1995; pour flûte de bambou, sanxian, zheng et pipa] - mais celte dernière œuvre est surtout jouée dans le monde chinois, c'est dommage. 
Nicolas Donin - On a pu récemment entendre San Xiao interprété par l'Ensemble instrumental Tianyin à Paris, le 5 février, au festival Présences 2002. Le $1^{\text {er février, }}$ les mêmes interprètes avaient donné le même programme à Pékin (Beijing Concert Hall). Cette pièce ne peut pas avoir le même sens dans les deux cas : en France, les instruments ne sont pas familiers, même s'ils sont mis en regard avec le répertoire de la musique traditionnelle. À votre avis, l'écoute de cette œuvre peutelle être complètement différente d'un côté et de l'autre? Est-ce la même œuvre?

Chen Qigang - Rien que pour l'instrumentation, le traitement des instruments traditionnels, on n'avait jamais écrit des choses comme ça en Chine - du moins à ce que je sache.

C'est très difficile à jover, ça va très vite, à la fois pour chaque instrument et pour la coordination de type "musique de chambre" (sans chef). Lors des premières répétitions, il y a cinq ans, la difficulté leur semblait presque insurmontable; aujourd'hui, tous les élèves l'apprennent, on essaie de l'imiter pour l'écriture de musique de chambre, c'est à la mode. Mais musicalement, c'est une pièce facile à comprendre, aussi bien pour les Chinois que pour les Français. Ce qui m'intéresse c'est surtout le timbre que je peux tirer de celte formation.

Nicolas Donin - La pièce serait donc aussi éloignée de la culture française là cause des instruments) que de la culture chinoise (à cause de la manière dont vous les faites sonner).

Chen Qigang - Oui, en quelque sorte. Mais pour les Chinois, cette formation est déjà bizarre : mettre ensemble ces quatre instruments est très rare. Et pour les Français, c'est plutôt le timbre qui surprend; quand Marc-André Dalbavie a entendu ça, il a dit: "C'est presque un son traité par informatique. "Pour moi, c'est une pièce que j'ai pu écrire sans presque aucun effort, en un mois; c'était comme un essai, un jeu. Mais avant cela, j'avais beaucoup discuté avec chaque instrumentiste pour connaître à fond chaque instrument et ajouter un peu plus de difficulté à ce qui m'était montré - afin qu'il y ait un enjeu pour l'interprète, l'envie de dépasser son niveau.

Nicolas Donin - Votre démarche dans San Xiao rend presque inopérante l'idée de mélange, et est par ailleurs bien distincte de la coexistence des contraires chère à Takemitsu; pensez-vous que ce soit une voie d'avenir? Un répertoire autonome pourraitil voir le jour?

Chen Qigang - Ça dépend, il faut une vision plus globale et plus historique. Je crois que le monde est dominé par l'argent, par l'économie. Si l'Europe a pu être le centre culturel du monde pendant quelques siècles, c'est parce qu'elle s'est développée en premier. Si la Chine reste toujours dans le tiers-monde, sans avoir une influence comparable à celle qu'ont les États-Unis économiquement et politiquement, leur culture ne pourra être exportée comme l'est la culture japonaise ou celle des États-Unis. J'ai discuté avec Zhang Yimou, le réalisateur d'Épouses et concu- 
bines" ${ }^{3}$, et il partage le même avis : "Si notre film n'entre pas dans le domaine commercial, dans le système d'Hollywood, il n'aura jamais assez de succès pour que les gens sachent seulement qu'il existe. » La musique chinoise, sans se mélanger avec la musique occidentale, ne pouvait pas se répandre facilement.

Nicolas Donin - Et vous vous dites qu'elle le pourra par elle-même désormais?

Chen Qigang - $\|$ y a une chance. Je crois qu'il y a une chance [...]

Nicolas Donin - Quel lien y a-t-il entre Un temps disparu - le concerto pour erhu et orchestre qui va être donné en première audition à Montréal les 23 et 24 avril 2002 - et Reflet d'un temps dispari ${ }^{4}$ ?

Chen Qigang - C'est une histoire triste - je veux dire du point de vue compositionnel. Je faisais grand cas de cette pièce; je tenais à ce qu'elle soit particulièrement réussie, puisque destinée à un très bon orchestre /l'Orchestre symphonique de Montréal) et une très bonne instrumentiste (Ma Xianghua). Charles Dutoit et moi avons découvert Ma Xianghua à Pékin; dès qu'on l'a vue, on a décidé de faire quelque chose ensemble. J'avais l'idée de composer spécialement pour cet instrument el pour cette instrumentiste; mais une fois que j'ai commencé à écrire, j'ai vu que c'était d'une difficulté inimaginable. Écrire pour un instrument si simple, avec un ambitus très restreint ( 2 octaves et demie), dont la note la plus grave est le ré une quinte plus haut que le violon, et dont la plus haute se situe une octave plus bas que le violon! En outre il y a une façon d'interpréter bien particulière, puisqu'il n'y a pas de touche et que la corde est suspendue - elle ne vibre donc pas naturellement: pour donner une sensation de vibration proche du violon, il faut appuyer extrêmement fort. Ca change la hauteur du son, et le vibrato est très lent. Par ailleurs, j'ai voulu, comme d'habitude, avoir une grande richesse d'harmonies en rapport avec le son orchestral; or cet instrument a un son très en dehors, donc la combinaison est délicate. Et puis je voulais varier les modes ef les tonalités, sans me borner au pentatonique; or les mutations sont impossibles. Enfin, j'aime beaucoup les sons harmoniques; mais la corde du erhu est très courte... [il sort un erhu et en jove pour illustrer son propos]. J'aime particulièrement les sons harmoniques lâchés, dans lesquels on laisse résonner le son - il y en a souvent dans le concerto pour violoncelle. Comme l'archet est enserré entre les deux cordes accouplées du erhu, on ne peut par définition pas le lâcher, il étouffe le son. Dans le même ordre d'idées, les pizzicati sont possibles mais très secs, très maigres, on ne les entend presque pas. J'ai donc essayé de m'adapter à l'instrument, mais le résultat était entièrement tonal et pour l'orchestre, cela ressemblait à un accompagnement de variété, une chanson arrangée. Cela, je n'aime pas non plus le faire. Pendant plus de trois mois, j'ai
3. Chen Qigang a écrit la musique de Raise the Red Lantern, le ballet conçu par Zhang Yimou d'après Épouses et concubines, et créé en mai 2001 à Pékin, Théâtre Tian Qiao.

4. Concerto pour violoncelle ef orchestre composé en 1995-1996 et créé le 23 avril 1998 au Théâtre des Champs-Élysées (Paris) par Yo Yo Ma et l'Orchestre National de France, sous la direction de Charles Dutoit. 
accumulé les brovillons, mais à la fin j'ai abandonné. J'ai écrit à Charles Dutoit pour lui expliquer la situation : si j'écris quelque chose de mauvais, ou quelque chose qui ressemble à une de mes œuvres précédentes - quel que soit l'instrument -, pour un créateur c'est néfaste; dans ce cas je préfère faire un arrangement. J'ai récrit entièrement la partie soliste du concerto pour violoncelle. Esthétiquement, ça reste la même œuvre - ainsi on ne peut pas dire que je me répète... mais pour le soliste c'est un très grand défi, il y a plusieurs passages beaucoup trop difficiles; je suis rentré spécialement à Pékin pour travailler avec Ma Xianghua, reprendre tous les traits, lui expliquer le sens musical de certains passages. Je lui ai demandé de jover comme un violoncelle, sans gonfler les sons à la façon traditionnelle mais en imitant le vibrato du violoncelle. C'est une création (périlleuse) pour tout ce qui touche l'écriture de l'instrument, son répertoire. C'est une Sequenza pour erhu! Si elle arrive à la jover, ça pourrait être fantastique.

Nicolas Donin - Restons dans l'actualité qui vous concerne en parlant d'Iris dévoilée, commande de la Fondation Koussevitzky et de Radio France qui vient d'être créée ${ }^{5}$...

Chen Qigang - Volontiers : j'ai reçu pas mal de réactions différentes, en général favorables - mais ceux qui sont contre ne me parlent pas... Pour moi personnellement, cette pièce est la conséquence de tout ce que j'ai fait depuis un certain temps ; j'ai décidé d'aller jusqu'au bout de ce que j'avais envie de faire, en n'étant pas contraint par quelque problème esthétique ou d'école que ce soit, en étant complètement libre.

Nicolas Donin - Certes, mais le contexte dans lequel l'œuvre était donnée n'était pas neutre : le fait de donner à la suite de votre création des œuvres néotonales ${ }^{b}$ permettait de percevoir clairement des parentés avec ce mouvement, controversé en France, qui n'est pas sans provoquer d'âpres discussions. Un voisinage avec Tan Dun aurait été beaucoup plus neutre. En ce sens, votre pièce est loin d'être absente de tout débat esthétique.

Chen Qigang - Dans ce concert du 6 février, entre la première et la deuxième partie, il y avait quand même un choc - même pour moi! Mais paradoxalement, quelqu'un comme Di Tucci, qui apprécie ma musique, je ne le connaissais pas; je l'ai rencontré à l'occasion de ce concert. Nos musiques restent très différentes. Quand je vois les discussions entre les néotonals et les soi-disant atonals, c'est pour moi un non-sens. Chacun est différent. La musique tonale peut être très mauvaise ou très bonne et la musique atonale aussi, ce n'est pas la question. Le problème pour moi, c'est la liberté individuelle: est-ce qu'on laisse les musiciens faire ce qu'ils veulent, sans établir de critère officiel (et ce critère officiel existe, même si on ne le dit pas)? J'aime que la France soit, comme toujours, un pays d'ouverture, un pays qui attire beaucoup de gens comme ce fut le cas à plusieurs périodes de son histoire.

Nicolas Donin - De ce point de vue, vous êtes français.

Chen Qigang - Oui.
5. Iris dévoilée, pour deux sopranos, une soprano de l'opéra de Pékin, pipa, zheng, erhu et grand orchestre. Présences 2002, concert du 6 février, Maison de Radio France, salle Olivier-Messiaen.

6. Arcanes symphoniques (CM) de Richard Dubugnon, Antarès de Jean-Jacques Di Tucci et Supernova de Guillaume Connesson composaient la deuxième partie du concert. 
Nicolas Donin - Et comment vous situez-vous par rapport à la nouvelle Chine, qui reste votre pays même si vous en êtes peutêtre moins proche aujourd'hui ? Pensezvous que vous retournerez vivre en Chine?

Chen Qigang - Je crois que la double culture est un avantage. Je suis heureux d'être chinois. Je suis content d'être en France. Ce sont deux grandes cultures.

Nicolas Donin - Il y a des gens qui n'ont pas fait le pas de la naturalisation, comme vous l'avez fait en 1992.

Chen Qigang - Le fait d'être naturalisé, c'était une simple nécessité pratique. Je ne pouvais pas vivre ici, et voyager en Europe, en tant que Chinois : pour aller en Angleterre, il me fallait demander le visa un mois à l'avance. La France fait partie de ces pays qui ont le privilège d'une grande liberté de circulation, dans la plupart des pays : j'ai pris le passeport correspondant! [...]

Nicolas Donin - Comment avez-vous abordé les Alla breve ${ }^{7}$ ? C'est un moyen de répartition pratique des commandes à des compositeurs nombreux et variés, mais par la force des choses, c'est peu à peu devenu un genre musical en soi : désormais, beaucoup de compositeurs français ont des pièces de $5 \times 2$ minutes dans leur catalogue...

Chen Qigang - J'y suis venu tout à fait par hasard. J'avais un très bon rapport avec l'Orchestre National de France. Depuis Présences 1996 [édition consacrée à la Chine], je désirais travailler pour la radio car je considère que c'est un moyen de diffusion très large ef plus démocratique que les autres (puisque le directeur change tous les six ans...). Quand on m'a proposé de faire un Alla breve, je me sentais moins jeune que les autres compositeurs à qui on le propose et je ne trouvais pas très valorisant qu'on me commande une œuvre sans me payer - ce fut ma première réaction. Plus tard j'ai eu un trou entre deux créations ef j'ai pensé accepter, en posant une condition : écrire non pour un petit effectif, mais pour l'Orchestre National. Après les réactions parfois très défavorables qui avaient suivi la création par ce même orchestre de Reflet d'un temps disparu, j'ai voulu prouver que Chen Qigang était l'un des meilleurs... Dans $W_{u} X_{i n g}{ }^{8}$, j'ai essayé de mettre le maximum de ce que je pouvais afin que la couleur sorte, que les moyens orchestraux soient poussés à plein régime. Je n'ai pas écrit ces petites pièces à la légère, pas du tout. Et grâce à Wu Xing, i'ai bénéficié d'une vaste diffusion, puis j'ai pu entrer en finale du Masterprize $2001^{9}$, ce qui $\mathrm{m}^{\prime} a$ permis de rencontrer un public et des interprètes encore plus nombreux.

Nicolas Donin - Encore une question de genre musical : dans Iris dévoilée, vous semblez recueillir le fruit de l'expérience de Wu Xing; vous décrivez des carac-
7. Émission hebdomadaire de France Musiques dont le principe est le suivant : une cuvre de $5 \times 2$ minutes est commandée à un compositeur et diffusée à cinq moments du lundi au vendredi à une heure de grande écoute, puis donnée intégralement la fin de semaine. Le compositeur est payé avec les droits d'auteur mais ne reçoit pas d'argent de la commande à proprement parler. La radio s'engage à diffuser la série deux fois dans la journée, et une fois sur Hector Iprogramme nocturne de France Musiques).

8. Wu Xing (1999) pour orchestre symphonique. Le titre signifie "Les Cinq Éléments»; l'œuvre est par conséquent composée de : "1. L'eau, 2. Le bois, 3. Le feu, 4. La terre, 5. Le métal.

9. Prix international organisé par la B.B.C. et décerné par le public sur internet; il y a eu deux éditions pour le moment (consulter, pour plus de précisions, le site http: //www.masterprize.com) 
tères, une mosaïque de pièces diverses traitant d'un aspect de la féminité lles titres des numéros sont : " 1 . Ingénue, 2. Pudique, 3. Libertine, 4. Sensible», etc.). Estce une sorte de cantate profane? Ou un double triple concerto (trois voix et trois instruments) - mais dont l'élément de virtuosité aurait été mis en retrait? Avez-vous délibérément brouillé les pistes?

Chen Qigang - J'ai voulu profiter de toutes les couleurs offertes par cet effectif important, mais en traitant la voix comme un instrument. La voix est seulement un timbre, c'est pourquoi les sopranos chantent souvent assises, mêlées à l'orchestre. Il fallait éviter que l'auditeur soit marqué par l'image de la personne, au lieu simplement d'entendre sa voix.

Nicolas Donin - Au contraire, quand une voix est soliste, vous la mettez en avant, et debout. La soprano classique et la chanteuse d'opéra de Pékin sont placées en vis-à-vis de part et d'autre de l'orchestre et intervertissent leurs places au milieu de l'œuvre. Quel est le sens de ce dispositif?

Chen Qigang - C'est le côté théâtral. La chanteuse traditionnelle est d'abord d̀ gauche, ensuite à droite; pour moi, le son qui vient de la droite est toujours plus lointain - je ne sais pas pourquoi. Le son à gauche, à côté des premiers violons, est donc plus proche; j'ai mis la présence à gauche et le lointain - la résonance à droite. Tantôt c'est l'Occident qui est présent et l'Orient qui répond; tantôt c'est l'inverse. Mais cette idée de changer la direction du son est secondaire, elle ne m'est venue qu'après la composition. Tant que la scène, la disposition réelle des musiciens n'étaient pas là, je ne savais pas comment faire. La veille de la première répétition j'ai discuté avec le chef : où place-ton les musiciens? Comment éviter des pertes de temps dans leurs déplacements? C'est là qu'on a donné forme à des choses qui étaient déjà présentes dans la musique.

Nicolas Donin - Le seul moment où la femme occidentale et la femme orientale se rejoignent, c'est quand elles hurlent, dans "Hystérique ". Cela m'a beaucoup intrigué...

Chen Qigang - "Hystérique" correspond à mon expérience personnelle, aux femmes que j'ai vues ainsi, incontrôlables, insupportables; ce sont des moments difficiles, ça casse tout... et ça casse la pièce, aussi. À ce momentllà, les trois voix n'étaient pas suffisantes, c'est pourquoi j'ai demandé à toutes les femmes de l'orchestre de crier. D'une manière générale, dans Iris dévoilée, je poursuis un travail sur la voix qui a commencé dans le ballet Épouses et concubines. Dans celui-ci, j'ai utilisé pour la première fois la voix traditionnelle féminine ( $c^{\prime}$ était déjà $\mathrm{Ma}$ Shuai qui chantait). C'était fantastique; elle était dans la tosse avec l'orchestre, et des gens m'ont dit "Qu'est-ce que c'est que ce son? ce n'est pas un basson... ", et ils percevaient progressivement une voix humaine. Pour moi ce fut une expérience très riche. Voilà ce qui m'a amené à choisir les différentes voix d'Iris dévoilée. Mais plus profondément, il s'agissait de réunir mes intérêts pour les timbres orchestraux ef le mélange des cultures, et mon désir de m'exprimer par moi-même, 
en décrivant ce monde féminin indispensable à l'homme. J'ai été assez audacieux pour cette pièce, en utilisant souvent un langage tout à fait tonal; c'est-à-dire que je ne pouvais pas m'arrêter d'écrire - parfois ça devenait très "Messiaen » aussi. Ce sont des influences marquées, je n'ai pas voulu m'en débarrasser ${ }^{10}$; ces mélodies-là sont peut-être ma faiblesse. On verra : dans un certain temps, disons cinq ans, peutêtre que je détesterai cela, mais pour le moment j'ai aimé le faire. [...] Avec cette pièce, il se peut que j'aie commencé à m'intéresser à l'opéra - à travers l'expérience de très grandes voix, et de ces mélanges avec les instruments; je vais peutêtre développer ces dimensions.
10. L'œuvre est dédiée "d̀ mon maître Olivier Messiaen $\$$ 


\section{«Une calligraphie est pour moi comme une partition inédite." "}

\section{Entretien avec Wen De-Qing.}

La musique de Wen De-Qing est le fruit d'une posture d'étrangeté. À son arrivée en Europe, face au monde de la musique contemporaine occidentale comme à celui de la langue française, le compositeur s'est trouvé très soudainement submergé d'informations, au point de devoir s'inventer - et de conserver par la suite - une écoute partielle, très ouverte mais immédiatement sélective, toujours à mi-chemin entre le jugement de goût et la radiographie analytique. Les ensembles instrumentaux occidentaux sont devenus, comme il le souhaitait, la référence sonore principale de sa pensée, mais dans une totale anhistoricité; car Wen ne recherche pas une historicisation du matériau inscrivant d'emblée l'acte créateur dans un hic et nunc socio-historique, en considérant le caractère rétrospectivement unique de chaque style musical du passé. Assez peu sensible à l'exigence avant-gardiste de n'aimer et de ne produire que du nouveau, il ne considère pas non plus la musique comme la manipulation d'un matériau déjà mort car toujours déjà disponible; à l'inverse de cette altitude postmoderne "constructiviste", Wen donne la priorité à une conception plus globale de l'œuvre, et du projet empirique qui lui donne consistance. De sorte que le hiatus entre culture chinoise (histoire de la musique et folklore paysan) et culture occidentale, qui me frappe toujours en tant que thème principal de sa production musicale, n'a en fait plus vraiment de sens pour lui - comme on le verra dans cet entretien. On pourrait croire, par suite, que la relative polyvalence stylistique décelable dans sa production récente (du style de quatuor bartókien à l'influence de la musique spectrale) n'est pas assumée comme telle par le compositeur. Au contraire, il semble plutôt la revendiquer. La recherche de la disparité - ou plutôł du "changement" pour reprendre son expression - apparaît parfois dans son discours comme un impératif moral.

Né en 1958 à Shuiji (Fujian), Wen De-Qing a quitté la Chine pour la Suisse en 1991 (peu après les événements de la place Tien An Men [1989]). II a étudié à Lyon avec Gilbert Amy (1993-1994), et en Suisse pendant plusieurs années. D'où la singularité d'une trajectoire d'émigration par laquelle la rupture, si elle intervient aussi autour de la trentaine, n'a pas lieu dans le courant des années 1980, comme c'est le cas pour Chen Qigang. À cet égard, l'un des intérêts de cet entretien était de rentrer dans le détail de certaines expériences fondatrices pour le compositeur, qu'il s'agisse de musique reçue ou conçue. Les frontières ou les ruptures ne se sont pas toujours là où on pourrait le croire; pour comprendre cela, il faut opérer des déplacements mentaux que le mouvement dialogique du questionnement met parfois clairement en évidence. 
Cet entretien a été réalisé à Paris, les 4 et 6 février 2002.

Nicolas Donin - Vous souvenez-vous de la première fois que vous avez entendu de la musique?

Wen De-Qing - Oui, des chansons, quand j'étais tout petit - vers six ou sept ans, pendant la Révolution culturelle. Je suis né dans un petit village où il n'y avait presque pas de musique. C'était l'époque du maoisme : tous les étudiants, à la sortie du collège, devaient partir aux champs, à la ferme, afin d'apprendre quelque chose des paysans. Ils amenaient un peu de musique, du violon chinois notamment; c'est la première fois que j'ai écouté ce genre de musique. Ils jouaient des airs de propagande maoïste ou de la musique russe, pour se distraire, mais pas vraiment de musique traditionnelle. Comme mon village était assez retiré et que la musique traditionnelle était interdite, je ne pouvais pas en entendre. II faut ajouter à cela une expérience antérieure : ma grande sœur avait un violon chinois qu'elle avait reçu d'un cousin, et je lui avais demandé de me donner une leçon. Nous portions tous un badge de Mao auquel on tenait beaucoup; je lui proposai de payer ma première leçon avec ce portrait, ce qu'elle accepta. Par la suite, j'ai joué du violon en cachette, essayant de restituer des mélodies entendues à la radio ou ailleurs.

Nicolas Donin - Et vous souvenez-vous de la première fois que vous avez entendu de la musique occidentale?

Wen De-Qing - C'est quand je suis entré à l'École normale supérieure du Fujian, au département de musique. Là, c'est la première fois que j'ai écouté un vieux 78 tours, du Beethoven - la Cinquième. L'enregistrement était de très mauvaise qualité, mais quand même... touchant.

Nicolas Donin - Dans quelles circonstances ça s'est passé exactement? C'était un professeur qui passait ce disque ou c'était vous qui vouliez écouter cette musique?

Wen De-Qing - C'est moi qui l'avais emprunté à la bibliothèque. Il n'y restait pas grand-chose, alors : partitions, disques, presque tout avait été brûlé. L'École venait de rouvrir, après dix ans de fermeture; c'était deux ans après la mort de Mao. Tout le monde pouvait à nouveau passer le concours; c'est cette année-là que je suis allé à l'École normale et que j'ai pu écouter ce disque.

Nicolas Donin - Lorsque vous êtes allé à l'École normale, c'était dans l'intention d'enseigner la musique traditionnelle ou dans celle qu'un jour, vous vous intéresseriez à la musique occidentale, à l'écriture occidentale?

Wen De-Qing - Vous savez, à cette époque-là, quand on venait d'une petite ville, on n'avait pas beaucoup de choix. À la réouverture de l'École, les candidats de dix années se sont présentés, il y avait beaucoup de concurrence : dans ma région, une personne sur cent pouvait être acceptée. Et il ne s'agissait encore que de l'École de ma région, on ne pensait pas à Pékin - c'était trop loin. On était dans ce coin, et la question n'était pas de vouloir étudier la musique traditionnelle, mais seulement de vouloir apprendre quelque chose en rapport avec la musique. 
Je ne pensais pas à la musique occidentale dont je ne savais rien, sinon quelques chansons soviétiques...

Nicolas Donin - À quel moment avez-vous eu des contacts plus fréquents avec la musique occidentale au point de vouloir la travailler?

Wen De-Qing - Mon professeur officiel s'intéressait surtout à la mélodie. Mais il y avait un autre professeur qui composait des symphonies, des concertos, avec des formations occidentales: Guo Zu-Rong. Chez lui, il y avait beaucoup de disques, surtout de musique russe, comme Tchaïkovsky, des choses comme ça. On en écoutait chez lui, où il faisait des cours d'analyse pour quelques élèves à titre particulier. C'est là que j'ai entendu beaucoup de Beethoven, je m'en souviens très bien : l'Opus 13, des sonates qu'il analysait magnifiquement. Premier thème, deuxième thème, comment développer le matériau, c'était la première fois que l'on me parlait de cela. En 1979, j'ai commencé à travailler avec lui et j'ai écrit mes premières pièces, notamment des Préludes pour piano qui ont même été publiés.

Nicolas Donin - De quels styles ces premières pièces étaientelles inspirées ? Quel langage parlaientelles?

Wen De-Qing - C'était déjà un peu debussyste! Car ce professeur essayait toujours de sortir de la tonalité, par exemple par la polytonalité... Mais c'est beaucoup plus tard que j'ai travaillé avec Luo Zhong-Rong à Pékin : son professeur avait été l'élève $\mathrm{d}^{\prime} H$ indemith, et lui-même avait traduit la théorie d'Hindemith depuis l'anglais. II m'a ouvert les portes de la nouvelle musique, c'était en 1987-1988. À cette époque, à Pékin, j'ai pu entendre des cassettes de Stravinsky, Penderecki, et quelques autres.

Nicolas Donin - Eł aujourd'hui, ces deux mondes sonores séparés /chinois traditionnel et occidental), est-ce dans votre musique que vous arrivez à les rassembler? Ou est-ce que vous pensez différemment à la musique chinoise d'une part, et à l'occidentale de l'autre?

Wen De-Qing - Mais déjà, à l'École normale, on devait apprendre la musique classique chinoise et la musique occidentale, ça pouvait se mélanger. Quand je suis sorti de l'École, j'ai enseigné deux ans dans la région d'où je suis originaire, puis j'ai travaillé pendant cinq ans dans une région au nord-ovest (Ningxia), où on entendait beaucoup de musique folklorique, ce qui $\mathrm{m}^{\prime}$ a considérablement influencé. Dans les pays communistes, l'État demandait souvent aux artistes de s'adresser au peuple en retournant à la tradition. Et encore aujourd'hui, en Europe, si l'on prend les pays de l'Est, le rapport des compositeurs avec le public y est plus étroit. Voyez Górecki, par exemple, ou Schnittke, ou encore Arvo Pärt - des compositeurs dont le style est moins complexe, plus près du public. Les Chinois ont donc eux aussi été influencés par cette attitude, à travers les figures de Béla Bartók et de Chostakovich. Pour moi, les deux mondes se sont mélangés et ne sont plus vraiment séparés. Naturellement, en vivant ici, i'ai été davantage imprégné de musique occidentale, mais la base, la pensée viennent toujours de la culture chinoise, de la philosophie chinoise. 
Nicolas Donin - C'est pour cela que vous choisissez souvent des titres chinois pour des œuvres dont l'écriture paraît surtout occidentale?

Wen De-Qing - Oui, au sens où il s'agit de produire quelque chose qui soit créé réellement par moi. Quand je suis arrivé ici, je comprenais très difficilement le français! J'étudiais aux conservatoires de Lyon et de Genève, mais je ne comprenais pas grand-chose, ni la première ni la deuxième année; j'étais donc obligé d'ouvrir les yeux, de chercher moi-même les partitions contemporaines, d'écouter les œuvres nouvelles au concert sans contextualisation. Et le fait de ne pas tout bien comprendre était sans doute bénéfique, cela me contraignait à clarifier mon point de vue, à trouver des idées personnelles. Par exemple, dans ma première pièce écrite en Europe, De la neige en été pour violon et grand orchestre, j'ai utilisé des quarts de tons et une série de 24 notes. Le dodécaphonisme me proposait douze notes... et j'en ai ufilisé vingtquatre. En ce qui a trait aux inversions, rétrogrades et autres, je les ai repensés à partir du taoïsme. En effet, certains textes taoïstes (notamment ceux qui décrivent les recettes d'immortalitél sont strictement inintelligibles si on les lit dans le sens habituel, de haut en bas ou de droite à gauche: ils cachent le sens de la lecture selon certaines règles, certaines clés. Si vous trouvez la première phrase, vous pouvez progressivement tout décrypter, mais ce n'est jamais linéaire - il y a un code secret et pour l'obtenir, vous devez vraiment patienter, vraiment aimer, avoir vraiment envie de devenir immortel. Pour ma part, j'ai pris une mélodie chinoise, j'al enlevé les notes qui se répétaient, j'ai modifié certaines hauteurs, un quart de ton plus haut ou un quart de ton plus bas, je les ai combinées de diverses manières pour obtenir $24 \times 4=96$ notes. J'ai constitué un tableau contenant toutes les transpositions : un mur entier avec 2264 notes! Puis j'ai déterminé certains sens de lecture, du centre vers les bords, ou en croisant les diagonales, etc. Et ainsi j'ai pu m'enlever de la tête les mélodies qui y existaient déjà.

Nicolas Donin - C'était pour les supprimer, les effacer?

Wen De-Qing - Pour les supprimer. Un peu au hasard, mais aussi avec certaines règles. Ainsi je pouvais me détacher de la façon dont j'aurais spontanément imaginé des mélodies. Voilà quelle méthode j'ai utilisée pour cette œuvre. Mais j'essaie de former un projet pour chaque pièce; à chaque fois bien sûr, il faut régler avec l'oreille, s'adapter à ce que l'instrument peut faire.

Nicolas Donin - Quel compositeur citeriez-vous en référence pour le face-à-face entre Chine et Occident, ou éventuellement entre Orient et Occident? Par exemple, dans le souffle, vous utilisez le $Y_{i}$ Ching : je ne peux éviter d'évoquer la manière dont John Cage s'en est servi.

Wen De-Qing - Je dirais plutôt que ce qui me sert de référence, c'est le dynamisme ef le sens très vivant de la construction logique d'un Bartók, ainsi que son rapport à la musique traditionnelle...

Nicolas Donin - Donc à votre avis, ce qui compte c'est qu'un compositeur ait réussi à réunir l'énergie de la musique populaire ef la musique savante, et non pas l'«Orient» et l'«Occident» ou la "Chine» et l'«Europe». 
Wen De-Qing - C'est ça. Orient ou Occident, ce n'est vraiment pas très important : la musique, c'est fait pour être écouté. Ce qui n'empêche pas qu'on entende très clairement dans ma musique certaines couleurs, certaines percussions, cerłaines mélodies, certains principes narratifs, certains types de contrastes spécifiquement chinois. En outre, la musique de John Cage n'était presque pas diffusée en Chine quand i'y étais. |Alors que j'ai été très marqué, au début des années 1980, par un pianiste américain qui était venu jover dans mon école des pièces de George Crumb - c'était mon premier contact avec la musique contemporaine. Le piano amplifié, l'idée de jover à l'intérieur du piano, j'avais trouvé cela magnifique.) Dans ma pièce Divination, i'ai utilisé le $Y_{i}$ Ching d'une façon complètement différente de celle de Cage. Je l'ai fait parce qu'il y a 64 degrés différents qui mènent de la Terre au Ciel de façon extrêmement progressive, en inversant le rapport du yin et du yang. Au départ, pour la Terre il y a six traits, tous yin; j'ai fait correspondre des intervalles à ces éléments. Par exemple, la seconde mineure est yin. J'ai utilisé ce principe pour classer le matériel intervallique. l'évolution est très continue : à chaque degré, un trait change. Lors de la première mutation, lorsqu'un trait devient yang et que les cinq autres restent yin, j'introduis une seconde majeure. Ainsi j'évite d'utiliser des procédés occidentaux de développement impliquant des répétitions thématiques ou harmoniques. lci, il y a un développement graduel logique qui correspond bien au sens du titre du Yi Ching : Le livre des mutations. Pour moi, comme pour le taoïsme, si rien ne change, c'est la mort. J'ai entendu bien des pièces de compositeurs "parisiens" dans lesquelles une seule idée ou une couleur est exploitée dans toute la pièce, ce qui permet une grande unité de caractère; pour moi, i'ai besoin de mouvement, de changement irréversible.

Nicolas Donin - De quelle manière le choix des instruments a-t-il une influence sur le projet de la pièce? En général dans une commande, ce qui est donné, c'est l'instrumentation. Avez-vous des idées qui préexistent aux instruments, ou bien partezvous de ceux-ci?

Wen De-Qing - Certes, il faut connaître le caractère de chaque instrument, savoir composer pour chacun d'eux... Mais cela ne doit pas m'empêcher d'introduire telle ou telle idée dans le dispositif, dans cette formation qu'on m'a imposée, que je n'ai pas choisie. Ainsi, dans Ji I et Ji / pour piano, i'ai recherché le son du gin "l, et le pianiste doit jouer à l'intérieur du piano - non pas à la façon de Crumb, mais avec des harmoniques et des glissandi sur une seule corde. Autre exemple : dans une commande d'un trio (très occidental : violon, violoncelle et piano), j'ai introduit des éléments de musique mongole en considérant le trille comme un thème. Dans mon premier quatuor à cordes, les instrumentistes doivent changer leur archet pour un plectre dans le deuxième mouvement. Cela fait référence à la grande importance des instruments à cordes pincées dans la musique chinoise (pipa, qin, zheng, etc.), en comparaison avec l'hégémonie des cordes frottées en Occident. Et dans le premier mouvement, inspiré de la notion taoïste de vide et par l'idée de Lao-Tseu selon laquelle la grande musique n'a pas besoin de beaucoup de notes, j'ai utilisé seulement quelques harmoniques. Pour ce faire, j'ai puisé dans mon expérience de la
1 1. Cithare chinoise à sept cordes, l'un des principaux ef des plus anciens instruments traditionnels. 
musique paysanne. J'ai travaillé des années avec les paysans, après le lycée; j'ai joué du violon chinois avec eux. Ils n'ont jamais mis les pieds dans un conservatoire, ils sont tous autodidactes et ne trouvent pas les notes justes facilement. Ils commencent donc par chercher doucement l'intonation, ce qui provoque un glissando avec harmonique, sur un quart de ton ou un sixième de ton; et une fois la note trouvée, ils appuient bien fort pour la garder! J'ai repris à mon compte cette technique en l'exagérant. Ainsi, chaque note est ornementée au début et à la fin [il chante des exemples], et acquiert une sonorité particulière.

Nicolas Donin - Avez-vous rencontré des problèmes avec les interprètes européens, par exemple en Suisse, pour la restitution de certaines techniques de jeu ainsi transférées? Je pense également aux différentes œuvres où vous faites parler, crier en chinois les instrumentistes.

Wen De-Qing - Bonne question! Bien sûr - d'autant que la musique chinoise n'est pas encore beaucoup jouée en Europe. Je prépare les créations avec les interprètes, et je m'inspire de la notation très précise des techniques telle qu'on la trouve dans de nombreuses partitions. Un jour, j'ai demandé au violoncelliste de l'ensemble Contrechamps, qui préparait un concert avec des œuvres de Tan Dun et de Brian Ferneyhough, quelle différence il voyait entre la préparation des différentes œuvres. II $\mathrm{m}$ 'a répondu que le Ferneyhough était très clair [sic], que tout était bien expliqué, alors qu'on manquait cruellement d'informations pour la musique de Tan Dun, ce qui rendait nécessaire la présence du compositeur. II y a là deux pensées traditionnellement un peu différentes; pour les Chinois, il y a de l'approximatif, de l'à-peu-près, chaque interprète a le droit d'improviser un peu ou d'inventer ses solutions. Pour ma part, je suis plutôt de l'avis "occidental » : il faut transmettre précisément son idée; mais c'est de plus en plus facile. En ce qui concerne la prononciation des cris, si je suis là, je peux donner quelques idées; si je ne suis pas là, ce n'est pas très important non plus, parce qu'il ne s'agit pas de comprendre le chinois, c'est impossible pour le public, donc c'est une question de couleur, de timbre, de sonorité. [...]

Nicolas Donin - Quand vous êtes venu ici, est-ce que vous êtes venu en Europe, en Suisse? ou en pays francophone? Où alliez-vous?

Wen De-Qing - Je suis venu à Genève (c'était la première łois que je quittais la Chine) grâce à un ami suisse, Pascal Schaer, qui avait fait des recherches en Chine sur la musique traditionnelle pour sa thèse de musicologie; il m'avait demandé des conseils car je $m$ 'intéressais moi aussi à certaines techniques $Y_{i}$ Chang de déclamation poétique - entre le chanté et le parlé -, et nous avions collaboré pour en enregistrer. Je lui avais fait entendre ma musique ef il m'avait conseillé de venir à Genève. 
Nicolas Donin - Si vous aviez eu le choix entre les divers pays d'Europe, auriezvous choisi la Suisse?

Wen De-Qing - Non. Depuis la Chine, on pensait toujours: Paris, ou bien Vienne, ou encore l'Allemagne. Bien sûr, beaucoup de compositeurs chinois sont allés aux États-Unis et au Canada. Mais j'apprécie la Suisse : j'y suis bien installé, elle m'a accordé un permis de séjour en tant qu'artiste, je reçois des commandes, il y a un bon ensemble, Contrechamps lalors qu'en Chine, à titre de comparaison, il y a quelques interprètes pour la musique contemporaine mais pas d'ensemble fixe).

Nicolas Donin - Vous avez sûrement des amis qui ont vécu une histoire assez comparable mais qui ont afterri à d'autres endroits...

Wen De-Qing - En effet. Tan Dun est aux États-Unis. Qu Xiaosong enseigne maintenant à Shanghai. Il y a Ye Xiaogang, Chen Yi... Plusieurs sont retournés en Chine, alors que Tan Dun ou Chen Yi sont restés aux États-Unis. En Europe, il y a Chen Qigang, Xu Shuya, ... On se connaît tous plus ou moins. En tout cas il me semble qu'il y a deux styles différents chez les compositeurs chinois, celui de ceux qui vivent en Europe et celui de ceux qui sont en Amérique. Ces derniers sont à la fois plus proches du public et plus liés aux contraintes commerciales, et leur musique est souvent moins "technique» que celle des musiciens qui ont étudié en Europe. [...]

Nicolas Donin - Vous pourriez rełourner vivie en Chine, aller aux États-Unis, etc. Pourtant vous choisissez de rester en Suisse?

Wen De-Qing - Désormais, en Suisse, j'ai un public! Pour le moment, je travaille à un opéra qui sera créé l'an prochain, ainsi qu'à un concert-portrait par l'Orchestre symphonique national chinois. Après, je ne sais pas si je retournerai en Chine, si je resterai en Suisse ou si je partirai ailleurs, mais tout ça n'est pas très important; le plus important, c'est de travailler, c'est d'écrire la meilleure musique possible.

Nicolas Donin - Maintenant j'aimerais parler calligraphie... Quel est son rôle dans vos différentes pièces revendiquant cette source d'inspiration? Dans Traces II pour ensemble, lorsqu'on regarde la partition, on voit bien qu'elle porte la marque d'une imitation graphique du tracé du pinceau, qui a des conséquences gestuelles ou mélodiques. Ce n'est pas vraiment le cas dans Traces III pour hautbois, en revanche. Quel modèle vous a fourni la calligraphie pour composer?

Wen De-Qing - La calligraphie est la source de l'art chinois. On peut faire une corrélation avec la musique chinoise, où il y a beaucoup de mélodie et peu d'harmonie. Tout à l'heure je parlais de mon premier quatuor et de la question de l'ornementation : dans la calligraphie d'un caractère, si vous voulez aller à droite, vous devez d'abord partir vers la gauche un petit peu; si vous voulez aller en bas, 
il faut d'abord monter un peu avec le pinceau. C'est une façon d'ornementer l'attaque de la note!

Par ailleurs, en calligraphie il existe plusieurs styles : I'un qui imite les classiques (écriture des sceaux), l'autre qui imite les caractères imprimés, réguliers (les élèves commencent par là), un autre encore, très rapide ef lié (écriture cursive), etc. En tout, on note a cinq styles principaux. De même, d'une composition à l'autre, je m'efforce de changer d'écriture, de techniques... D'ailleurs, écrire Traces I, II, puis III, c'était presque entrer dans la logique d'un cycle - ce qui signifierait que je dois encore en écrire deux!...

J'adore la calligraphie. C'est un art qu'on peut apprécier même sans comprendre le sens de ce qui est écrit. Les paysans changeaient chaque année les calligraphies devant leur porte, par goût - alors qu'ils ne savaient pas lire. C'est la force de l'abstraction. Même un lettré ne peut pas lire certains caractères tracés trop vite : ça n'a aucune importance, seule comple la beauté de la différence. Quand on apprend, on imite d'autres styles, mais il faut bientôt trouver son style propre. Une calligraphie (par exemple celle que j'ai placée en couverture de Traces III) est pour moi comme une partition inédite [il désigne des endroits sur l'image] : espace, son parfois un peu flou (avec beaucoup d'encre), parfois très lent, parfois très rapide. Il y a la construction, aussi : avant d'écrire un mot, vous devez imaginer ce que vous voulez faire, mais il n'est pas sûr que le résultat sera conforme. Parce que le pinceau est mou, ou à cause de la qualité de l'encre, des aspérités du papier.

Nicolas Donin - Ces surprises, comment les retrouvez-vous dans l'écriture de la pièce?

Wen De-Qing - C'est à travers la personnalité de l'interprète, qu'il soit bon ou mauvais, qu'on les retrouve. Dans Traces III, on joue parfois sans l'anche ou avec des bruits de clés; selon la facture de l'instrument aussi, tout peut changer. Pour produire les sons multiphoniques - que j'ai beaucoup utilisés dans les trois Traces - il y a beaucoup de notes, c'est très difficile : chacun a une technique un peu différente... et produit un son un peu différent. Le souffle compte beaucoup aussi. On peut obtenir un son multiphonique plus aigu et plus fort, mais moins riche - et vice versa.

Nicolas Donin - Tout à l'heure vous disiez que la calligraphie créait une structure - à la fois visuellement et dans le temps de l'écriture. C'est déjà de la composition. Je repense à ce que dit Donatoni dans le film introductif d'Alfred, Alfred (1998) : il relale une discussion entre sa femme et leur nouveau voisin. Ce dernier lui demande " $Q u^{\prime}$ est-ce qu'il fait, votre mari? » elle répond qu'il est compositeur. "Ah bon, compositeur? il travaille dans l'imprimerie? » Commentaire de Donatoni : ce voisin avait très bien exprimé - bien involontairement! - l'essence typographique de la composition telle que lui-même la pratique. Dans Traces II, 
on trouve parfois un assemblage/collage de matériau à la Donatoni; on a l'impression que vous juxtaposez des caractères musicaux. Est-ce le cas?

Wen De-Qing - On le voit déjà dans Traces I. Ou dans le solo de piano de Traces II, et dans d'autre passages. J'essaie de contourner ce qui ressemble à une forme $A B A$, dans laquelle les parties vont être sensiblement d'égale longueur et ordonnées de façon prévisible. Je cherche une grande disparité de pulsations : soudain un passage très rapide voire saccadé (changements internes de pulsation) succède à un temps étiré.

Nicolas Donin - Il y a une autre dimension de l'influence calligraphique qui articule - mais dans l'autre sens - temps vécu et composition visuelle. En effet, pour Traces I, il est prévu que vous exécutiez une calligraphie en temps réel, pendant que les interprètes jouent. Réalisez-vous toujours la même calligraphie? Y a-til un rapport entre le matériau musical et la calligraphie prise en référence?

Wen De-Qing - Je pense qu'on peut voir la musique. C'est pour cela qu'on a des concerts, on voit. Percevoir le geste avec le son, c'est très agréable. Si l'on veut isoler l'écoute, autant se passer un CD. Or, pour la calligraphie, on utilise un papier spécial : aussitôt que le pinceau touche le papier, il y a déjà des bruits spécifiques. De même quand on trempe le pinceau dans l'encre. Parfois le rythme de l'écriture convient à celui de la musique parfois il est contradictoire. Cela produit une sorie de polyphonie ou de polyrythmie. On peut alors trouver une relation entre les deux choses.

Nicolas Donin - Mais cette relation, est-ce vous qui la déterminez? Par exemple, en adaptant votre manière de peindre à ce que vous savez que les interprètes vont jover?

Wen De-Qing - Je choisis un poème à l'avance - pas toujours le même d'une fois à l'autre. De préférence un poème lié à la musique. Mais l'idée qu'on ferait la même chose chaque fois est illusoire, car il s'agit d'improvisation.

Nicolas Donin - Au fond, il y a deux choses très distinctes, et dont il importe qu'elles le restent : vous, vous calligraphiez; les interprètes jouent, et c'est au spectateur-auditeur de trouver par lui-même des relations.

Wen De-Qing - Je ne fabrique pas les relations. Mais parfois je les guide un peu, en choisissant de suivre la musique ou non - sur le plan de la vitesse notamment. Cette manière de procéder plaît souvent au public, en tout cas. Après chaque concert, des gens viennent vers moi : "J'aimerais bien avoir celte calligraphie s'il vous plaît. " Mais souvent c'est l'organisateur qui la garde... 
Nicolas Donin - Quelle importance attribuez-vous à la voix? Je pense en particulier à la pièce Complainte, pour voix et ensemble de percussions, qui est votre composition la plus fréquemment exécutée, mais aussi à l'opéra que vous commencez à composer cette année.

Wen De-Qing - La voix humaine est bien sûr très importante. Quand j'étais tout petit, j'avais remarqué que le son du violon chinois, que j'aimais tant, ressemblait à celui de la voix - une voix un peu nasillarde. J'ai beaucoup écrit pour instruments à cordes. Mais de toute façon, en Chine, écrire pour la voix est la base de l'apprentissage académique de la composition. Tous les jeunes compositeurs dojvent commencer par écrire des chansons; pour entrer à l'École normale, j'ai chanté devant le jury, en m'accompagnant au violon chinois, une chanson intitulée J'offrirai le meilleur thé à Mao. Tan Dun, qui passait le même concours à Pékin, avait composé quant à lui un Chant à la gloire de Mao. L'écriture pour la voix restait déterminante pendant toute la formation.

Dans Complainte, j'utilise une voix de style "opéra de Pékin», une voix d'homme chantant un rôle de femme, mais avec une puissance plus grande qu'un ténor. L'accompagnement requiert des «instruments" pauvres, qu'on trouve dans les poubelles (canettes vides, bouteilles de verre...), dans une cuisine (casseroles, etc.) - dans la vie quotidienne. II n'y a donc sur la scène aucun instrument normal, si l'on peut dire. Il s'agit de garder en mémoire et de restituer la charge émotionnelle d'un événement traumatique. Certains objets ont une valeur symbolique autant que musicale, comme les chaînes de fer. Les instruments classiques étaient insuffisants pour évoquer l'inoubliable. II fallait un moyen d'expression spécifique, inusité. Les bidons d'essence, les couvercles de casseroles, les marmites ont un son très violent. Les bouteilles, les verres de cristal ont un son très doux. Il y a aussi du papier qu'on déchire, des paquets de biscuits, des boîtes à thé, trois tailles de bocaux pour poissons rouges, cinq bols japonais, etc. Par-dessus tout cela, une voix très aiguë qui crie souvent. Cela constitue une sonorité unique, et d'ailleurs je n'ai écrit qu'une seule pièce dans ce style. Les hauteurs n'étant pas fixes, je me réfère à des hauteurs relatives et travaille beaucoup sur l'organisation du rythme. Ce dernier organise les chiffres de 1 à 9 sous forme de carré magique. Certains taoïstes utilisaient ce carré pour expliquer le $Y_{i}$ Ching. Qu'on additionne une ligne, une colonne ou une diagonale, on obtient toujours 15 :

$\begin{array}{lll}4 & 9 & 2 \\ 3 & 5 & 7 \\ 8 & 1 & 6\end{array}$

Horizontalement, il y a trois percussionnistes avec trois rythmes différents. Verticalement, cela donne toujours 15 notes. On a l'impression que rien n'est ensemble, que la vitesse change sans cesse; c'est très tendu, alors que la métrique demeure stable. J'ai chanté cette pièce à Darmstadt en 1998 avec l'atelier de per- 
cussion dirigé par Mircea Ardeleanu et, depuis, il la joue un peu partout ; c'est lui qui chante la partie soliste, il a même endisqué la pièce au Brésil!

Nicolas Donin - Donc celte pièce n'est pas forcément liée à votre voix, à votre façon de restituer ces sentiments personnels.

Wen De-Qing - Non. D'autant que le texte est métaphorique; il s'agit de Enfants, où êtes-vous cachés? de Wang Li Hua, poétesse taïwanaise. Même si ma familiarité avec l'œuvre est insurpassable par définition, j'aime que d'autres se l'approprient. Mais un chanteur de l'opéra de Pékin n'est pas nécessairement le plus à même d'y parvenir, j'ai déjà eu l'occasion de le vérifier. En effet, j'utilise seulement certaines techniques de l'opéra de Pékin, et je les modifie; il me faut une voix de contre-ténor, tel type de glissando, la connaissance des quatre tons du chinois et de quelques caractéristiques du style d'opéra de Pékin, et une bonne dose d'enthousiasme. Le reste est affaire de concentration lors de l'exécution.

Nicolas Donin - Comptez-vous intégrer ce type de vocalité dans votre opéra Le pari, qui sera créé en septembre 2003 au festival Amadeus (Genève)?

Wen De-Qing - Quelques éléments seulement. Je retravaillerai surtout sur les quatre tons de la langue chinoise, en les exagérant, en les ornementant, etc. Les tons peuvent être entendus comme des embryons de mélodie. Après avoir considéré la voix de diverses manières - aussi bien en écrivant des chansons traditionnelles qu'en étudiant la technique de déclamation Yin Chang -, après des années d'écriture de musique d'ensemble, je me sens prêt aujourd'hui à créer un opéra de chambre chanté en chinois. 


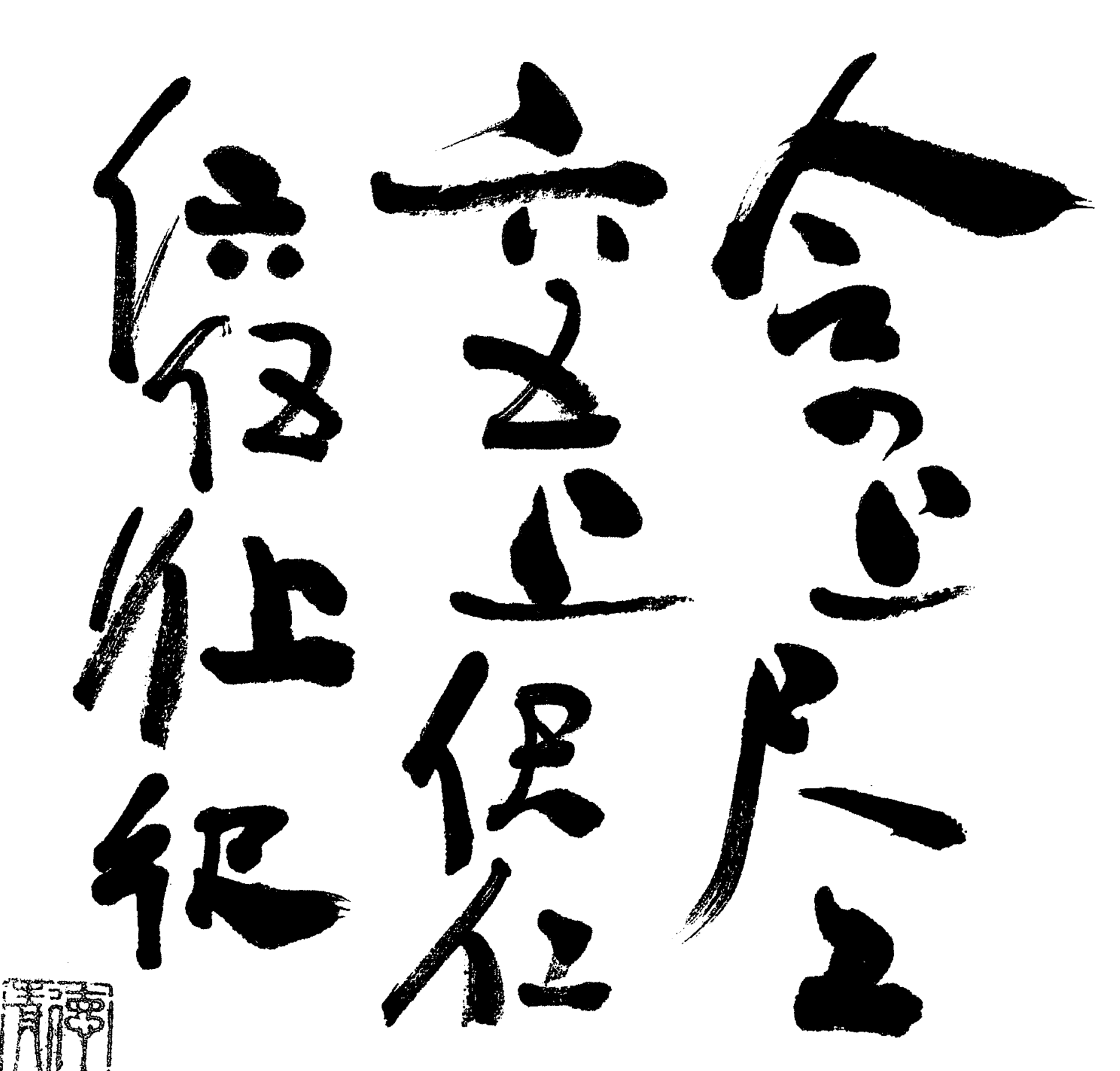

